Image 2:034

\title{
Advances in Cytoarchitectonic Mapping of the Human Amygdala and the Hippocampus
}

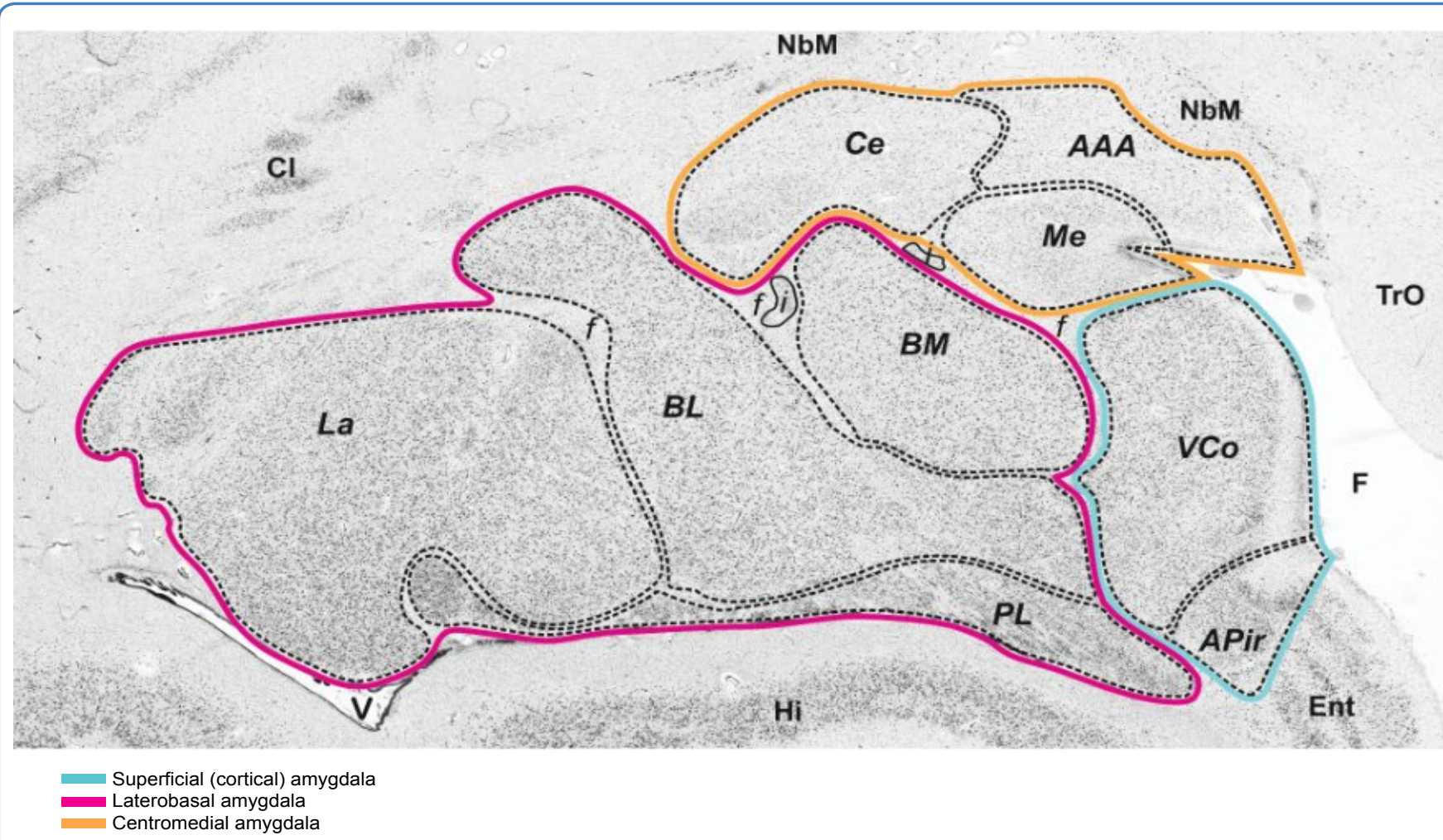

Figure 1: Cytoarchitecture of the amygdala and neighboring cortical and subcortical structures in a coronal section of a human postmortem brain. The centromedial nucleus is labelled by an orange line and the basolateral complex by a red line. The VCo belongs to the superficially located part of the amygdala. AAA anterior amygdaloid area, APir amygdalopiriform transition area, BL basolateral nucleus, BM basomedial nucleus, BV basoventral nucleus, Ce central nucleus, f fiber bundles, i intercalated islands, La lateral nucleus, Me medial nucleus, PL paralaminar nucleus, VCo (ventral) cortical nucleus. Neighbouring structures: Cl Claustrum, Ent entorhinal cortex, F endorhinal sulcus, Hi hippocampus, NbM Nucleus basalis of Meynert, TrO Tractus opticus, V lateral ventricle.

Source: Figure 1 (minimally altered) "Springer Anat Embryol, Cytoarchitectonic mapping of the human amygdala, hippocampal region and entorhinal cortex: Intersubject variability and probability maps, 210, 2005, 343-352, Amunts K, Kedo O, Kindler M, Pieperhoff P, Mohlberg H, Shah NJ, Habel U, Schneider F, Zilles K CSpringer Verlag, "With permission of Springer".

\section{Information}

\section{Olga Kedo ${ }^{1 *}$, Karl Zilles ${ }^{1,2,3}$ and Katrin Amunts ${ }^{1,2,4}$}

${ }^{1}$ Institute of Neuroscience and Medicine (INM-1), Research Centre Jülich, Germany

2JARA-BRAIN, Jülich-Aachen Research Alliance, Germany

3Department of Psychiatry, Psychotherapy and Psychosomatics, RWTH Aachen University, Germany

${ }^{4} \mathrm{C}$. and O. Vogt-Institute for Brain Research, HeinrichHeine-University Düsseldorf, Germany

*Correspondence: Olga Kedo, Institute of Neuroscience and Medicine (INM-1), Research Centre Jülich, 52425 Jülich, Germany; E-mail: o.kedo@fz-juelich.de
Citation: Kedo O, Zilles K, Amunts K (2016) Advances in Cytoarchitectonic Mapping of the Human Amygdala and the Hippocampus. Clin Med Img Lib 2:034

Published: March 23, 2016

Copyright: () 2016 Kedo O, et al. This is an open-access content distributed under the terms of the Creative Commons Attribution License, which permits unrestricted use, distribution, and reproduction in any medium, provided the original author and source are credited. 


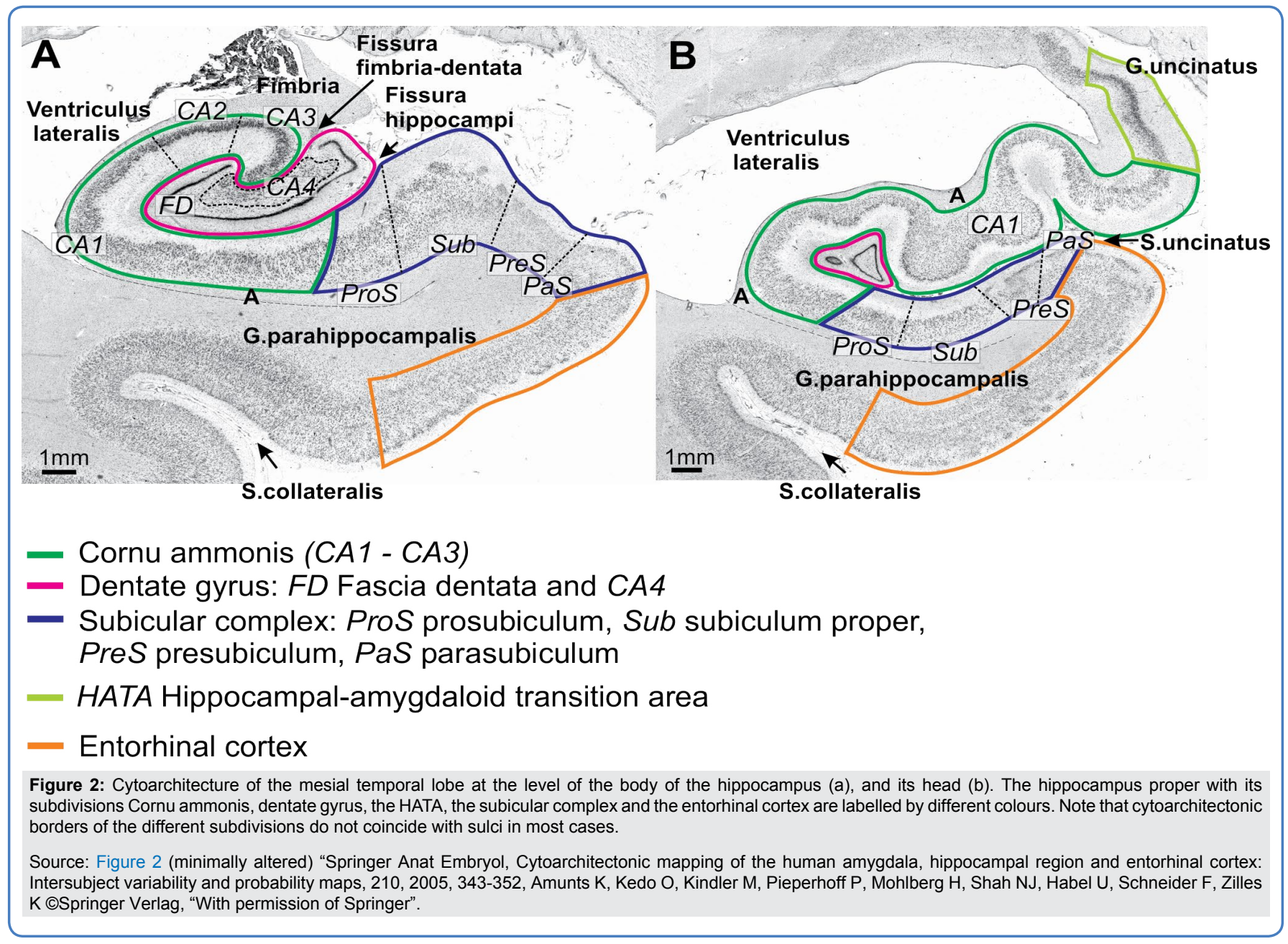

\section{Keywords}

Amygdala, Hippocampus, Cytoarchitecture

\section{Discussion}

The human amygdala and hippocampus are key structures for emotion and memory processing. They are involved in various neurological and psychiatric disorders. Since each of both brain regions is structurally and functionally extremely segregated [1], their numerous subdivisions are implicated to different degrees in specific pathological processes, e.g. epilepsy [2,3].

Although modern functional imaging techniques can assign dysfunctions to the whole amygdala or hippocampus after specific impairments [4], the disorder- or lesion-specific involvement of the clearly defineable, and functionally as well as structurally diverse cytoarchitectonic subdivisions remains to be elucidated. Therefore, cytoarchitectonic probability maps are a prerequisite for an anatomically sufficiently precise localization of functional imaging data [1]. This was realized by registration of probability maps of the subdivisions of the amygdala and hippocampus to a stereotaxic reference space, which is also used for the localization of functional imaging data. E.g., a prevailing contribution of the basolateral amygdala was revealed by using this combined approach in impaired acquisition of conditioned fear in patients with Urbach-Wiethe disease [5].

Here we present further advanced cytoarchitectonic parcellations of the amygdala (Figure 1) and the hippocampal formation (Figure 2). The cytoarchitectonic identification of the subdivisions of the hippocampus and amygdala in histological sections is the basis of the finegrained probability maps, as well as of volumetric measurements.

\section{References}

1. Amunts K, Kedo O, Kindler M, Pieperhoff P, Mohlberg H, et al. (2005) Cytoarchitectonic mapping of the human amygdala, hippocampal region and entorhinal cortex: Intersubject variability and probability maps. Anat Embryol 210: 343-352.

2. Yilmazer-Hanke DM, Wolf HK, Schramm J, Elger CE, Wiestler OD, et al. (2000) Subregional pathology of the amygdala complex and entorhinal region in surgical specimens from patients with pharmacoresistant temporal lobe epilepsy. J Neuropathol Exp Neurol 59: 907-920.

3. Graebenitz S, Kedo O, Speckmann EJ, Gorji A, Panneck H, et al. (2011) Interictal-like network activity and receptor expression in the epileptic human lateral amygdala. Brain 134: 2929-2947.

4. Adolphs R, Tranel D, Buchanan TW (2005) Amygdala damage impairs emotional memory for gist but not details of complex stimuli. Nat Neurosci 8: 512-518.

5. Klumpers F, Morgan B, Terburg D, Stein DJ, van Honk J (2015) Impaired acquisition of classically conditioned fear-potentiated startle reflexes in humans with focal bilateral basolateral amygdala damage. Soc Cogn Affect Neurosci 10: 1161-1168. 